\title{
РАЗВИТИЕ МЕТОДА ЛЮМИНЕСЦЕНТНОГО КОНТРОЛЯ СОСТАВА ПЛАЗМЫ И ОБРАБАТЫВАЕМОЙ ПОВЕРХНОСТИ В ТЕХНОЛОГИИ АНТИКОРРОЗИОННОЙ ЗАЩИТЫ НЕФТЕГАЗОВОГО ОБОРУДОВАНИЯ
}

\author{
Тюрин Юрий Иванович', \\ tyurin@tpu.ru \\ Ван Яомин', \\ jldxwym@mail.ru \\ Сыпченко Владимир Сергеевич', \\ sypchenkov@mail.ru \\ Никитенков Алексей Николаевич', \\ nik@tpu.ru \\ 1 Национальный исследовательский Томский политехнический университет, \\ Россия, 635050, г. Томск, пр. Ленина, 30.
}

Актуальность. Надежность и целостность нефтедобывающего оборудования, сроки его эксплуатации обеспечиваются комплексом мер по борьбе с коррозией, в частности использованием технологий газопламенного напыления защитных покрытий на проектируемые или восстанавливаемые детали. В данных технологиях эффективным методом контроля за состоянием состава плазмы и качества напыляемой поверхности может служить явление гетерогенной хемилюминесценции. Гетерогенные хемилюминесцентные реакции обладают селективностью и высокой чувствительностью к типу поверхности и сорту возбуждающего газа. Использование оптических методов для изучения, контроля и управления в неравновесных системах газ-твердое тело открывает новые аналитические и аппаратурные возможности в физике поверхности, химии, плазмохимии, технологии полупроводников и люминофоров, в решении экологических проблем. Изучение процессов адсорбции-десорбции, диссоциации, диффузии, рекомбинации газовых частиц, дефектообразования и роста кристаллической решетки с использованием явления гетерогенной хемилюминесценции является актуальной задачей физики конденсированного состояния. Поскольку явление гетерогенной хемилюминесценции реализует возможности осуществления селективных экспресс-методов анализа при простом аппаратурном оснащении с пределом обнаружения свободных атомов, радикалов, примесей в газовой фазе и в составе поверхностных слоев конденсированных сред до $10^{-6} \%$ (мол).

Цель: исследование процессов в неравновесных системах газ - твердое тело и определение параметров этого взаимодействия на основе регистрации характеристик гетерогенной хемилюминесценции; разработка нестационарных методов определения параметров взаимодействия газ-твердое тело с использованием явления гетерогенной хемилюминесценции, контроль параметров газовой среды и состояния поверхности конденсированных сред.

Объекты: атомно-молекулярные пучки водорода, кристаллофосфор ZnS-Mn², приповерхностные области взаимодействия газ - твердое тело.

Методы: методы, основанные на явлении гетерогенной хемилюминесценции в атомарном водороде для определения скоро-

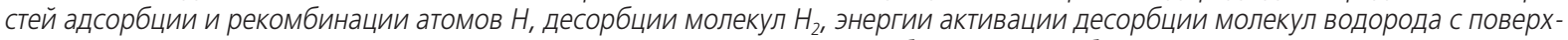
ности $\mathrm{ZnS}-\mathrm{Mn}^{2+}$. Методом "темновой» паузы определена скорость рекомбинации адсорбированных атомов водорода по механизму Лэнгмюра-Хиншелвуда.

Результаты. Выполнено сравнительное исследование люминесценции ZnS-Mnn ${ }^{2+}$ при возбуждении светом (фотолюминесценции) и атомарным водородом (гетерогенной хемилюминесценции). Изучены спектрально-кинетические характеристики люминесценции. Установлены механизмы и параметры взаимодействия атомов водорода с поверхностью сульфида цинка (сечения, частотные факторы, энергии активации) с использованием спектрально-кинетических характеристик гетерогенной хемилюми-

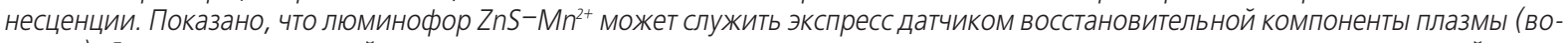
дород). Явление гетерогенной хемилюминесценции составляет основу оперативных методов контроля начальных стадий модификации поверхности твердых тел в процессах пучково-плазменной обработки материалов.

\section{Ключевые слова:}

Нефтегазовое оборудование, коррозия, защитные покрытия, газоплазменное напыление, фотолюминесценция, гетерогенная хемилюминесценция, поверхность, атомы водорода, нестационарные процессы, взаимодействия атомов водорода с поверхностью.

\section{Введение}

Увеличение сроков службы нефтедобывающего оборудования во многом определяется комплексом мер для борьбы с коррозией металла при его физико-химическом взаимодействии с окружающей средой [1]. Повышение ресурса оборудования и сооружений может быть достигнуто использованием технологий газоплазменного напыления защит- ных покрытий на поверхность защищаемых или восстанавливаемых деталей [2]. Эффективным методом контроля состава плазмы и качества напыляемой поверхности может служить явление гетерогенной хемилюминесценции (ГХЛ) [3].

Хемилюминесценция продолжает оставаться областью активных исследований [4], что связано с разнообразием её практических применений. Яв- 
ление ХЛ заключается в прямом преобразовании энергии возбужденных состояний, образованных свободными атомами и промежуточными радикалами, в электромагнитное излучение [5-10]. Возбужденные частицы могут дезактивироваться и при передаче энергии люминофорам с относительно высоким квантовым выходом [10]. Тем самым ХЛ служит основой перспективной техники с повышенной надежностью, быстрым откликом, экономически эффективным инструментарием и простым управлением [11]. ХЛ с повышенными пределами обнаружения [10, 12] становится чувствительным аналитическим методом [12-20].

Среди многочисленных направлений исследования ХЛ систем быстро развивающейся областью стала гетерогенная хемилюминесценция (ГХЛ) $[3,21]$. ГХЛ возбуждается в актах рекомбинации свободных атомов тепловой энергии на поверхности люминофоров. Свободные атомы взаимодействуют с самым первым слоем поверхности. Выделяющаяся энергия рекомбинации $\left(\mathrm{H}+\mathrm{H} \rightarrow \mathrm{H}_{2} 4,5\right.$

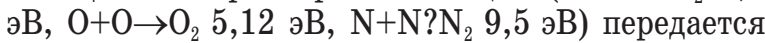
непосредственно поверхностным и приповерхностным центрам свечения (10-40?) и приводит к избирательному возбуждению люминесценции приповерхностной области.

Распространение методов ГХЛ на газовую фазу с твердофазной диагностикой и обнаружением связано с высокой интенсивностью сигналов, селективностью и другими уникальными свойствами ГХЛ [21, 22]. Реализованы способы определения концентрации свободных атомов $\mathrm{H}, \mathrm{O}, \mathrm{N}$, а также молекул $\mathrm{SO}_{2}, \mathrm{CO}_{2}$ в вакууме и газах по интенсивности ГХЛ детектора. Подбором селективного кристаллофосфора-излучателя для данной смеси газов удается достичь уровней интенсивности ГХЛ, дающих соотношение свечения анализируемой примеси к фону, равное $10^{2}$ и более [22-28]. Метод эффективен для определения $\mathrm{SO}_{2}$ в газовых смесях, находящихся при пониженных давлениях, а также для анализа воздуха при относительно больших концентрациях $\mathrm{SO}_{2}$, например, в отходящих газах металлургических производств [26, 27]. Предел обнаружения $\mathrm{SO}_{2}$ равен $\left(10^{-2}-10^{-4}\right) \%$ мол или $10^{-2} \mathrm{Mr} / \mathrm{m}^{3}$, люминофор $\mathrm{Y}_{2} \mathrm{O}_{2} \mathrm{~S}-\mathrm{Eu}$. В случае $\mathrm{CO}_{2}$ атмосферы интервал определения концентраций может быть расширен от $5 \cdot 10^{-3}$ до 50 \% мол. Предельная обнаруживаемая относительная концентрация $\mathrm{CO}_{2}$ составляет $10^{-3} \%$ мол, люминофор $\mathrm{CaCO}_{3}-\mathrm{Bi}$ [22, 28]. АО «ОПТЭК», г. Санкт-Петербург, разработало серию газоанализаторов, аттестованных в уполномоченной организации при Агентстве по охране окружающей среды США (ЕРА) и одобренных EPA в качестве средств измерения озона для задач мониторинга атмосферного воздуха, промышленных газовых выбросов, воздуха рабочей 30ны, транспортных (автомобильных) выбросов, технологических газовых смесей. В основу работы анализаторов положен эффект гетерогенной хемилюминесценции, возникающей в результате реакции $\mathrm{O}_{3}, \mathrm{H}_{2} \mathrm{~S}, \mathrm{SO}_{2}, \mathrm{NO}, \mathrm{NO}_{2}, \mathrm{NH}_{3}, \mathrm{CO}, \mathrm{CO}_{2}$ с окисля- емыми химическими веществами композиции [29].

Полупроводниковый материал $\mathrm{ZnS}$ является прямозонным соединением группы AII-BVI $[30,31]$ с оптической шириной запрещенной зоны 3,41-3,91 эВ. Сульрид цинка используется для создания люминофоров $\mathrm{ZnS}-\mathrm{Ag}$ с синим цветом свечения ZnS-Ag 450, (Zn,Cd)S-Ag 5 - кинескопы, и рентгеновские трубки с зелёным цветом свечения. Соединения $\mathrm{ZnS}-\mathrm{Cu}$ - используются для светящихся табло, панелей, как люминофоры трубок осциллографов, в ультрафиолетовых светодиодах, как эффективные люминофоры на плоских дисплеях, в фотоэлектрических устройствах. $\mathrm{ZnS}-\mathrm{Mn}^{2+}$ оранжевый, $\mathrm{ZnS}-\mathrm{Mn}^{2+}, \mathrm{Te}^{2+}$ (750 нм) и $\mathrm{ZnS}-\mathrm{Sn}^{2+}$, (715 нм) красный и т. д. [32, 33].

Настоящая статья посвящена исследованию ГХЛ ZnS-Mn ${ }^{2+}$. Люминофор $\mathrm{ZnS}-\mathrm{Mn}^{2+}$ имеет потенциальное применение в полевых эмиссионных устройствах (FED) [34]. Легированные полупроводниковые наночастицы $\mathrm{ZnS}-\mathrm{Mn}^{2+}$ используются в качестве люминофоров, а также в тонкопленочных электролюминесцентных устройствах [35]. Эффективность свечения люминофоров увеличивается с уменьшением размера частиц, и люминесцентные материалы на основе $\mathrm{ZnS}$ используются в дисплеях приборов в качестве датчиков, в лазерах и т. д. $[36,37]$. ГХЛ облегчает быструю идентификацию и обнаружение широкого класса соединений с применением высокоэффективных твердотельных люминофоров, к которым относится $\mathrm{ZnS}-\mathrm{Mn}^{2+}$.

Среди люминесцентных методов изучения элементарных актов взаимодействия газ-поверхность следует выделить исследования, основанные на нестационарных измерениях [38]. В их основе лежит регистрация люминесцентного отклика системы на изменение одного из внешних параметров: плотность потока атомов и молекул, температура образца, темновая пауза, фотоподсветка и пр., определяющих интенсивность ГХЛ. С одной стороны, изменение внешних параметров должно быть осуществимо экспериментально, а с другой чтобы их изменение приводило к такому люминесцентному отклику, по характеру которого удавалось бы определять параметры процессов взаимодействия газ - твердое тело. Спектр люминесценции несет наиболее детальную информацию о структуре центра свечения и его ближайшем окружении. ГХЛ связана с возбуждением приповерхностной области кристалла атомами и молекулами тепловой энергии. Симметрия кристаллического поля и колебательный спектр поверхности отличаются от объемных. Это приводит к различию спектров при оптическом и химическом способах возбуждения. Дополнительные изменения в спектрах поверхностной люминесценции обусловлены деформацией (перестройкой) решетки и существованием специфических поверхностных центров свечения.

Сравнительное исследование спектров ГХЛ и фотолюминесценции (ФЛ) служит информатив- 
ным методом изучения механизмов возбуждения ГХЛ получения сведений:

- о параметрах и динамике кристаллической решетки;

- структуре и пространственном распределении центров свечения, примесей и дефектов;

- процессах изменения свойств поверхности при воздействии активных газов;

- изменении симметрии кристаллического поля при переходе от объема к поверхности;

- частотах колебаний поверхностных мод кристалла и локальных колебаний адсорбата и пр.

Выбор активатора, служащего спектроскопическим зондом, определяется целью исследований. Ионы с незаполненными $d$-оболочками $\left(\mathrm{Mn}^{2+}\right)$, сильно взаимодействующие с решеткой, могут служить индикаторами динамических свойств поверхности. Ионы, способные образовывать центры свечения при ассоциации с со активаторами, вакансиями или при сегрегации примеси, позволяют судить о перераспределении введенных в образец атомов активатора с температурой и изменении состава поверхности в атмосфере активного газа. Спектроскопическим зондом может выступать и сама адсорбированная частица.

\section{Техника эксперимента}

Установка для измерения спектрально-кинетических характеристик люминесценции кристаллофосфоров при разных условиях и способах возбуждения, в том числе пучком атомарного водорода, разработана и изготовлена в отделении экспериментальной физики инженерной школы ядерных технологий Томского политехнического университета и подробно описана в [38].

Установка состоит из следующих основных частей:

- вакуумная часть;

- система нагрева и контроля температуры;

- источник особо чистого молекулярного и атомарного водорода (высокочастотный генератор плазмы);

- система подачи газа;

- системы регистрации интенсивности свечения;

- системы колориметрической и люминесцентной регистрации атомав $\mathrm{H}$.

Предельное остаточное давление в системе $\sim 10^{-6}$ торр; рабочее давление определяется скоростью напуска газа (через натекатель марки DFDZ$750)$ в высокочастотный (ВЧ) генератор плазмы и составляет $1-10^{-3}$ торр.

Атомарный водород, взаимодействующий с поверхностью образца, получается с помощью без электродного емкостного высокочастотного разряда в молекулярном водороде (ВЧ-генератора УВЧ 30-2). Для определения изменений концентрации атомов водорода в вакуумной ячейке использован метод изотермического калориметра с мостом Уинстона с вольфрамовой нитью в качестве прецизионного датчика в плече [39].

Молекулярный водород получается электролизом дистиллированной воды в генераторе водорода и кислорода (ГВЧ-12М1). Используемый в ГВЧ12M1 метод очистки водорода основан на использовании нанотехнологических полимерных мембран, селективно проницаемых по водороду. Водород хранится в баллонах и напускается в камеру по системе для напуска газа. Скорость напуска газа контролируется управляющим компьютером.

\section{Результаты и обсуждение}

Спектры люминесценцииZnS-Mn2+

$\mathbf{Z n S}-\mathbf{M n}^{2+}$. В решетку ZnS марганец входит в виде ионов $\mathrm{Mn}^{2+}$, заменяющих ионы $\mathrm{Zn}^{2+}$ в узлах решетки. Кубическая решетка $\mathrm{ZnS}-\beta$ (ссралерит) относится к группе пространственной симметрии $\mathrm{T}^{2}, \mathrm{ZnS}-\alpha$ (вюрцит) к $\mathrm{C}_{6}^{4}$. Локальная симметрия кристаллического поля, в месте нахождения парамагнитного иона $\mathrm{Mn}^{2+}$ в $\mathrm{ZnS}-\beta$, относится к группе правильного тетраэдра, а в ZnS- $\alpha$ - к группе тетраэдра, искаженного вдоль тригональной оси. В кристаллическом поле симметрии $\mathrm{T}_{d}$ происходит расщепление первого электронно-возбужденного состояния иона $\mathrm{Mn}^{2+}:{ }^{4} \mathrm{G} \rightarrow{ }^{4} \mathrm{~T}_{1}+{ }^{4} \mathrm{~T}_{2}+\left({ }^{4} \mathrm{E},{ }^{4} \mathrm{~A}_{1}\right)$. В центрально-несимметричном поле кристалла состояния разной четности перемешиваются, четность перестает служить характеристикой отдельных уровней, и диагональные матричные элементы возмущения становятся для отдельных переходов отличными от нуля [40]. Этим, вероятно, объясняется возможность наблюдать интенсивную фотолюминесценцию $\mathrm{ZnS}-\mathrm{Mn}$ в марганцевой полосе, в отличие от ФЛ $\mathrm{CaO}-\mathrm{Mn}$.

На поверхности и в приповерхностной области симметрия окружения центра свечения $\mathrm{Mn}^{2+}$ понижается до $\mathrm{C}_{3 \mathrm{v}}$. В этом случае произойдет дополнительное расщепление уровней ${ }^{4} \mathrm{~T}_{1} \rightarrow{ }^{4} \mathrm{E}+{ }^{4} \mathrm{~A}_{2}$, ${ }^{4} \mathrm{~T}_{2} \rightarrow{ }^{4} \mathrm{E}+{ }^{4} \mathrm{~A}_{1}$. Излучательные переходы ${ }^{4} \mathrm{E} \rightarrow{ }^{6} \mathrm{~A}_{1}$, ${ }^{4} \mathrm{~A}_{1} \rightarrow{ }^{6} \mathrm{~A}_{1}$ дадут поляризованные линии в люминесценции - перпендикулярно и параллельно оси $\mathrm{C}_{3}$. Обрыв связей при образовании поверхности, наряду с понижением симметрии окружения центров свечения, находящихся на поверхности, изменит, в результате деформации решетки, величину кристаллического поля в приповерхностной области. В случае сжатия кристаллической решетки в направлении, перпендикулярном поверхности, произойдет усиление кристаллического поля лигандов. Усиление кристаллического поля приведет к длинноволновому смещению спектра излучения $\mathrm{Mn}^{2+}[41,42]$.

В приповерхностной области кристалла понижение симметрии можно рассматривать как возмущение, не приводящее к значительному расщеплению уровней ${ }^{4} \mathrm{~T}_{1}$ и ${ }^{4} \mathrm{~T}_{2}$.

Более сильное расщепление этих уровней соответствует понижению симметрии окружения от $\mathrm{T}_{d}$ до $\mathrm{C}_{3 \mathrm{v}}$ для ионов $\mathrm{Mn}^{2+}$, расположенных в плоскости поверхности.

Марганец оказался очень эффективным активатором при химическом возбуждении. Сечение возбуждения при рекомбинации атомов Н достигает $10^{-20} \mathrm{~cm}^{2}$. Благодаря этому предельно малые кон- 
центрации марганца $\left(10^{-6} \%\right)$ обнаруживают себя в спектрах свечения ГХЛ.

На рис.1 приведены спектры ФЛ и ГХЛ образца ZnS-Mn. Спектр ФЛ представлен оранжевой полосой с $\lambda_{\max }=585$ нм $\left({ }^{6} \mathrm{~A}_{1} \rightarrow{ }^{4} \mathrm{~T}_{1}\right)$ (кривая 1 , рис. 1, б). Небольшой пик проявился при 665,5 нм.

В спектре ГХЛ ряду с полосой 585 нм основной становится широкая полоса $\lambda_{\max }=665$ нм $(T=295 \mathrm{~K})$ (кривая 3 , рис. 1, б). В фосфорах, не содержащих марганец, полоса 665 нм отсутствует. Восстановление поверхности сухим водородом приводит к накоплению вакансий серы в приповерхностной области кристалла. Вакансии серы, понижая симметрию окружения иона $\mathrm{Mn}^{2+}$ с $\mathrm{T}_{d}$ до $\mathrm{C}_{3 V}$, увеличивают вероятность излучательного перехода ${ }^{4} \mathrm{E} \rightarrow{ }^{6} \mathrm{~A}_{1}$ в ионах активатора, расположенных у поверхности, и увеличивают интенсивность красной полосы.

Появлению красной полосы ГХЛ $\mathrm{ZnS}$ ? $\mathrm{Mn}$ в водороде также благоприятствуют высокие концентрации марганца ( $>0,5$ вес. \% ). За красную полосу $\Gamma_{\mathrm{X}} Л_{\mathrm{H}}$ ответственны либо центры, в которых ион $\mathrm{Mn}^{2+}$ ассоциирован с вакансией серы, либо комплексные центры, состоящие из двух и более ионов $\mathrm{Mn}^{2+}$. Образованию комплексных центров $\mathrm{Mn}^{2+}$ $V_{s}^{* * *}$ в силу принципа компенсации способствуют вакансии серы $V_{s}^{* *}$ (кривые 4,5 , рис. 1, б).

При сколе монокристаллов $\mathrm{ZnS}-\mathrm{Mn}$ (плоскость (011) и (120)) в вакууме или молекулярном водороде наблюдается лишь слабая вспышка свечения, затухающая за 0,5-5 с. Однако при сколе монокристаллов ZnS-Mn в атомарном водороде в момент скола происходит интенсивная вспышка свечения, достигающая величин $10^{12}$ квантов $/ \mathrm{cm}^{2} \mathrm{c}$ (ZnS-Mn). Люминесцентное свечение сравнительно медленно затухает и на образце $\mathrm{ZnS}-\mathrm{Mn}$ переходит в стационарное за время $\geq 10^{2}$ с [25].
Спектр свечения ZnS-Mn в атомарном водороде (рис. 1,8 ) включает основную полосу (содержится и в спектре ФЛ) $\lambda_{\max }=588$ нм дополнительные полосы ( $\left.\lambda_{1 \max }=665 \mathrm{Hм} \mathrm{и} \lambda_{2 \max }=705 \mathrm{Hм}\right)$, отсутствующие при ФЛ. С ростом температуры у данных полос наблюдается коротковолновое смещение, характерное для иона $\mathrm{Mn}^{2+}$ с незаполненной $3 \mathrm{~d}^{5}$ оболочкой. Основная полоса ГХЛ $\lambda_{\max }=588$ нм, как и при ФЛ, испускается ионами $\mathrm{Mn}^{2+}$, расположенными непосредственно у поверхности сульфида цинка. Полоса 705 нм испускается $\mathrm{Mn}^{2+}$-центрами, расположенными непосредственно на поверхности и ассоциированными с вакансией серы. Полоса 665 нм относится к $\mathrm{Mn}^{2+}$-центрам на поверхности, на которых адсорбированы молекулы $\mathrm{H}_{2}$.

Люминесцентные методы изучения взаимодействия атомов и молекул с поверхностью твердых тел

Кинетические и стационарные характеристики ГХЛ связаны как с состоянием поверхности твердого тела, так и с механизмами взаимодействия атомов и молекул с поверхностью, приводящими к возбуждению ГХЛ. Построение механизма ГХЛ основано на проведении комплекса экспериментальных исследований, когда параллельно измеряются люминесцентные, адсорбционные и электрофизические характеристики образца. Полученные результаты составляют основу для построения модели механизма возбуждения ГХЛ. Если модельные зависимости интенсивности люминесценции от времени, плотности потоков атомов и молекул, температуры образца качественно соответствуют эксперименту, то в этом случае можно так подобрать параметры модели, чтобы достичь и количественного совпадения. Решение обратной задачи позволяет найти сечения, энергии активации, частотные факторы, теплоты адсорбции атомов и молекул.

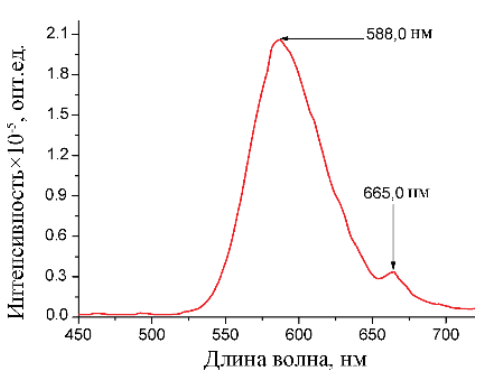

$a / a$

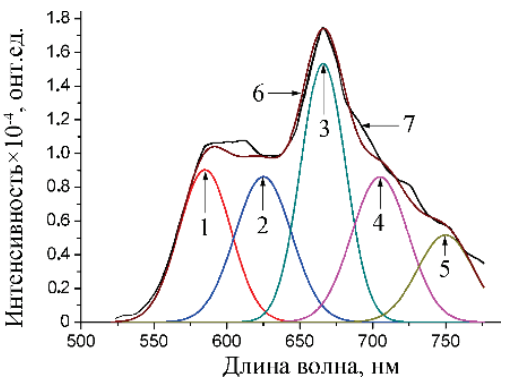

$\sigma / b$

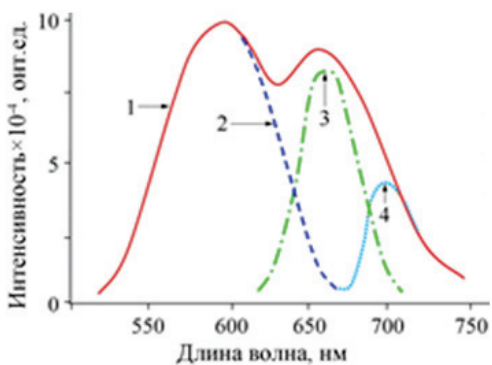

$B / c$

Puс.1. Спектры люминесценции $\mathrm{ZnS}-\mathrm{Mn}^{2+}$ (2,5 вес. \%), $295 \mathrm{~K}$ : а) возбуждение микрокристаллического образиа ртутной лампой (ДРТ 125-1) с фильтром (УФС-06) линиями 317, 339, 369 нл; б) возбуждение микрокристаллического образиа атомарныл водородом, кривые 1-6 результат разложения эксперилентальной кривой 7 методом Аленцева-Фока. Плотность потока атолов Н в эффузионном пучке $10^{17} \mathrm{~cm}^{-2} \mathrm{c}^{-1}$; давление $H+H_{2}$ в области высокочастотной разряда $1,3 \cdot 10^{-3}$ торр; в) после скола монокристалла ZnS-Mn2+ в атомарном водороде, кривые 2-4 результат разложения экспериментальной кривой 1 методом Алениева-Фока. Плотность потока атомов $Н$ в эффузионном пучке $10^{16} \mathrm{~cm}^{-2} \mathrm{c}^{-1}$; давление $\mathrm{H}+\mathrm{H}_{2}$ в области высоко частотной разряда $1,3 \cdot 10^{-3}$ mopp

Fig. 1. Luminescence spectra of $\mathrm{ZnS}-\mathrm{Mn}^{2+}$ (2,5 wt.\%), $295 \mathrm{~K}$ : a) microcrystalline sample excitation by (DRT 125-1) mercury lamp with (UFS-06) filter of 317, 339, $369 \mathrm{~nm}$ lines; b) microcrystalline sample excitation by atomic hydrogen, curves 1-6 are the results of experimental curve 7 decomposition by the Alentsev-Fock method. H atoms flux density in the effusion beam is $10^{17} \mathrm{~cm}^{-2} \mathrm{~s}^{-1} ; \mathrm{H}+\mathrm{H}_{2}$ pressure in the area of the high frequency discharge is $1,3 \cdot 10^{-3}$ torr; c) after cleavage of the $\mathrm{ZnS}-\mathrm{Mn}^{2+}$ single crystal in atomic hydrogen, curves $2-4$ are the result of the experimental curve 1 decomposition by the Alentsev-Fock method. $H$ atoms flux density in the effusion beam is $10^{16} \mathrm{~cm}^{-2} \mathrm{~s}^{-1} ; \mathrm{H}+\mathrm{H}_{2}$ pressure in the high frequency discharge is $1,3 \cdot 10^{-3}$ torr 
Наиболее доступными экспериментальными характеристиками РРЛ являются кинетические кривые разгорания ГХЛ и зависимости стационарной интенсивности от температуры образца и плотности потока атомов.

Механизм возбуждения ГХЛ включает следующий минимальный набор последовательно протекающих процессов [43]:

$$
\begin{gathered}
\mathrm{H}+\mathrm{L} \stackrel{v_{1}}{\longrightarrow} \mathrm{HL} \\
\mathrm{H}+\mathrm{HL} \stackrel{v_{2}}{\longrightarrow} \mathrm{H}_{2} \mathrm{~L} \\
\mathrm{H}_{2}+\mathrm{L} \stackrel{v_{-3}}{\longrightarrow} \mathrm{H}_{2} \mathrm{~L}
\end{gathered}
$$

Над стрелками указаны отнесенные к единице времени вероятности соответствующих реакций.

$$
\begin{gathered}
v_{i}=j \sigma_{i 0} \exp \left(-\frac{E_{i}}{k T}\right)=j \sigma_{i}, i=1, \\
v_{-3}=v_{-30} \exp \left(-\frac{q_{3}+E_{3}}{k T}\right) .
\end{gathered}
$$

Введены обозначения поверхностных концентраций в момент времени $t: \mathrm{L} \rightarrow N(t), \mathrm{RL} \rightarrow N_{1}(t)$, $\mathrm{R}_{2} \mathrm{~L} \rightarrow N_{2}(t)$.

Система кинетических уравнений для определения $N(t), N_{1}(t), N_{2}(t)$ имеет вид [43]

$$
\left.\begin{array}{l}
\dot{N}(t)=-v_{1} N+v_{-3} N_{2} \\
\dot{N}_{1}(t)=v_{1} N-v_{2} N_{1} \\
\dot{N}_{2}(t)=v_{2} N_{1}-v_{-3} N_{2}
\end{array}\right\} .
$$

Интенсивность люминесценции для произвольных начальных заполнений поверхности атомами и молекулами $N_{1}(0)=N_{10}, N_{2}(0)=N_{20}, N(0)=N_{0}-N_{10}-N_{20}$, равна:

$$
\begin{gathered}
I(t)=\eta v_{2} N_{1}(t), \\
N_{1}(t)=\frac{v_{1} N_{0}}{r_{1}-r_{2}}\left[A_{1} e^{r_{1} t}-A_{2} e^{r_{2} t}+C\right], \\
A_{1,2}=1+\frac{v_{-3}}{r_{1,2}}+\frac{\left(r_{1,2}+v_{-3}\right) N_{10}}{v_{1} N_{0}}-\frac{N_{20}}{N_{0}}, \\
C=v_{-3}\left(\frac{1}{r_{2}}-\frac{1}{r_{1}}\right), r_{1,2}=-\lambda\left(1 \mp \sqrt{1-\mu / \lambda^{2}}\right),
\end{gathered}
$$

При «малых» $t\left(\left|r_{1,2} t\right|<<1\right)$ интенсивность ГХЛ равна (1-3):

$$
I(t)=\eta v_{2} N_{10}+\eta\left[v_{1}\left(N_{0}-N_{10}-N_{20}\right)-v_{2} N_{10}\right] v_{2} t .
$$

Начальная вспышка свечения ГХЛ интенсивностью

$$
I_{0}=\eta v_{2} N_{10}
$$

связана с рекомбинацией свободных атомов с адсорбированными на поверхности в момент времени $t=0\left(N_{10} \neq 0\right)(4)$. После вспышки наблюдается линейный рост интенсивности ГХЛ со временем (рис. 2).

$$
\frac{d I(t)}{d t}=\eta\left[\sigma_{1}\left(N_{0}-N_{10}-N_{20}\right)-\sigma_{2} N_{10}\right] \sigma_{2} j^{2} .
$$

Кинетические кривые (3)-(5) разгорания ГХЛ

$$
I(t)=\eta v_{2} N_{1}(t)
$$

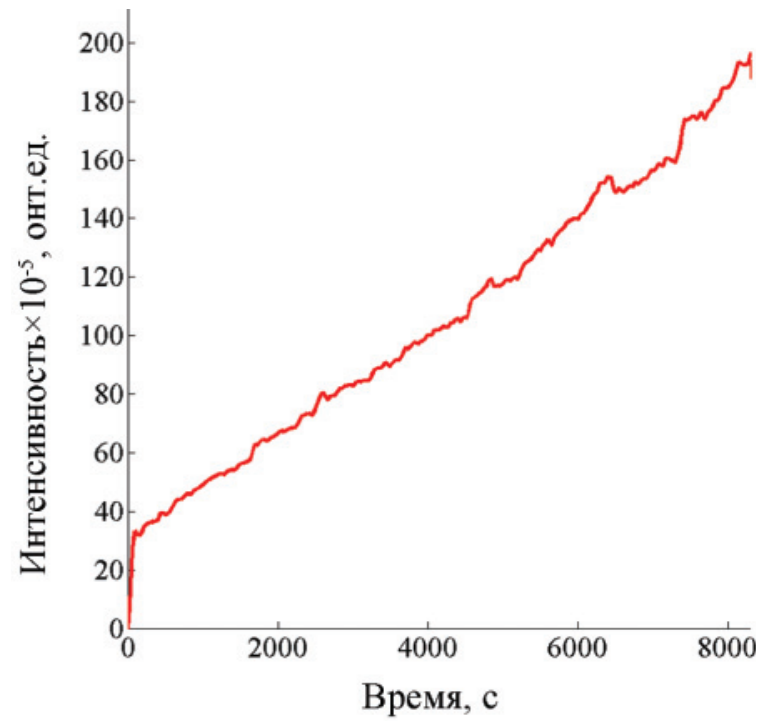

Pис.2. Зависилость интенсивности гетерогенной хемилюминесиентной фосфора $\mathrm{ZnS}-\mathrm{Mn}^{2+}$ от времени (при малых $<10^{-12} \mathrm{~cm}^{-3}$ концентрациях атомов водорода), $\mathrm{T}=295 \mathrm{~K}$

Fig. 2. Dependence of heterogeneous chemiluminescent intensity of phosphorus $\mathrm{ZnS}-\mathrm{Mn}^{2+}$ on time (at small $<10^{-12} \mathrm{~cm}^{-3} \mathrm{concen}$ trations of hydrogen atoms), $T=295 \mathrm{~K}$

в простейшем случае представляют немонотонную кривую с одним максимумом (рис. 3 , кривая 1 ). Но экспериментальные кривые значительно разнообразнее. Эти особенности связаны с процессами энергообмена в адсорбционном слое по мере накопления на поверхности адсорбата [44] и с процессами послойного травления поверхности ZnS атомарным водородом [45] (рис. 3, кривая 2).

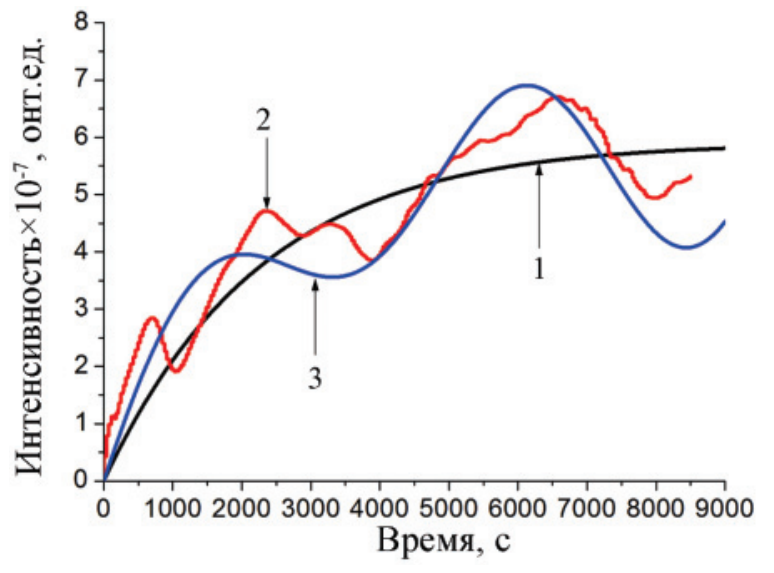

Pис.3. Люминесиенция $Z n S-M n^{2+}$ в атомарном водороде. $T=295$ K. Поверхность $\mathrm{ZnS}-\mathrm{Mn}^{2+}$ предварительно очищена прогревом образиа в вакууме $3,0 \cdot 10^{-6}$ торр: 1 - аппроксимация зависимостью (8); 2 - эксперимент; 3 - аппроксимация с учетом энергообмена в адсорбиионном слое [43]

Fig. 3. Luminescence of $\mathrm{ZnS}-\mathrm{Mn}^{2+}$ in atomic hydrogen. $\mathrm{T}=295 \mathrm{~K}$. The $\mathrm{ZnS}-\mathrm{Mn}^{2+}$ surface was previously cleaned by heating the sample in a vacuum of $3,0 \cdot 10^{-6}$ torr: 1 - approximation by dependence (8); 2 - experiment; 3 - approximation taking into account energy exchange in the adsorption layer [43]

Кинетическая кривая разгорания ГХЛ (рис. 3, кривая 1) описывается зависимостью: 
Таблица 1. Параметры $v_{1}, v_{2} u v_{-3}$ кинетической кривой 1, рис. 4

Table 1. Parameters $v_{1}, v_{2}$ and $v_{-3}$ of kinetic curve 1, Fig. 4

\begin{tabular}{|l|c|c|c|}
\hline Относительные вероятности/Relative probabilities & $v_{1}: \mathrm{H}+\mathrm{L} \stackrel{v_{1}}{\longrightarrow} \mathrm{HL}$ & $v_{2}: \mathrm{H}+\mathrm{HL} \stackrel{v_{2}}{\longrightarrow} \mathrm{H}_{2} \mathrm{~L}$ & $v_{-3}: \mathrm{H}_{2}+\mathrm{L}_{-3} \stackrel{v_{-3}}{\longrightarrow} \mathrm{H}_{2} \mathrm{~L}$ \\
\hline Значения, $\mathrm{c}^{-1} /$ Values, $\mathrm{s}^{-1}$ & $6,67 \cdot 10^{-5}$ & $4,25 \cdot 10^{-4}$ & $3,26 \cdot 10^{-4}$ \\
\hline
\end{tabular}

$I(t)=127,02 \exp (-0,00561 t)-$

$-127,87 \exp (-0,00564 t)+589,48$;

$$
(I-\text { отн. ед., } t-\mathrm{s}) \text {. }
$$

Поскольку разница между показателями степени $r_{1}$ и $r_{2}$ в (6), (7) меньше достижимой погрешности, следует перейти в (3) к пределу $\Delta r \rightarrow 0$ :

$$
\begin{gathered}
I(t)=\lim _{r_{1} \rightarrow r_{2}} \eta v_{2} \frac{v_{1} N_{0}}{r_{1}-r_{2}}\left[A_{1} e^{r_{1} t}-A_{2} e^{r_{2} t}+C\right]= \\
I_{0}+\left(I_{\infty}-I_{0}\right)\left(1-e^{r t}\right)+ \\
+I_{\infty} r t e^{r t}\left[\left(1+\frac{r}{v_{-3}}\right)+\frac{I_{0}}{I_{\infty}}\left(1+\frac{v_{-3}}{r}-\frac{N_{20}}{N_{10}}\right)\right] ; \\
r_{1,2}=-\lambda, 2 \lambda=v_{1}+v_{2}+v_{-3}, \\
\mu=\left(v_{1}+v_{2}\right) v_{-3}+v_{1} v_{2}, \quad \mu=\lambda .
\end{gathered}
$$

Параметры $v_{1}, v_{2}$ и $v_{-3}$ для уравнения (8) представлены в табл. 1 .

На рис. 4 приведена долговременная кинетика ГХЛ $\mathrm{ZnS}-\mathrm{Mn}^{2+}$. Изменение интенсивности свечения, вероятно, связано с последовательным послойным травлением поверхности $\mathrm{ZnS}$ атомарным водородом [45]. Начальная вспышка свечения отражает предварительное заполнение поверхности атомами водорода.

Нестационарные люминесцентные методы изучения взаимодействий атомов водорода на поверхности $\mathrm{ZnS}-\mathrm{Mn}^{2+}$

Люминесцентное свечение, возбуждаемое при взаимодействии свободных атомов и радикалов с поверхностью, позволяет изучать insitu, без внесения возмущений в систему, важные детали физико-химических процессов на поверхности в неравновесных и нестационарных условиях.

Примером исследований такого рода служит люминесцентный метод разделения ударного и диффузионного механизмов рекомбинации атомов водорода на поверхности $\mathrm{ZnS}-\mathrm{Mn}^{2+}[43,46]$ :

$$
\begin{gathered}
\mathrm{R}+\mathrm{RL} \stackrel{v_{2}}{\longrightarrow} \mathrm{R}_{2}^{\mathrm{v}} \mathrm{L}, \quad 2 \mathrm{RL} \stackrel{k}{\longrightarrow} \mathrm{R}_{2}^{\mathrm{v}} \mathrm{L}+\mathrm{L}, \\
\mathrm{R}_{2}^{\mathrm{v}} \mathrm{L}+\mathrm{Mn}_{\mathrm{s}}^{2+} \rightarrow \mathrm{R}_{2} \mathrm{~L}+\left(\mathrm{Mn}^{2+}\right)_{\mathrm{s}}^{*}, \\
\left(\mathrm{Mn}^{2+}\right)_{\mathrm{S}}^{*} \rightarrow \mathrm{Mn}_{\mathrm{S}}^{2+}+h v .
\end{gathered}
$$

Здесь звездочкой обозначено возбужденное состояние центра свечения $\mathrm{Mn}^{2+} \mathrm{S}$ на поверхности; $h v$ - испускаемый квант света; $v_{2}$-вероятность ударной рекомбинации в единицу времени; $k$ константа скорости реакции диффузионной рекомбинации атомов на поверхности.

Интенсивность люминесценции $I$ пропорциональна скорости рекомбинации атомов и квантовым выходам ГХЛ $\eta, \eta_{1}$ в реакциях ударной
$R+R L \stackrel{v_{2}}{\rightarrow} R_{2} L$ Ридила-Или (РИ) и диффузионной

$2 R L \stackrel{k}{\rightarrow} R_{2} L$ Лэнгмюра-Хиншелвуда (ЛХ) рекомбинации атомов.

$$
I=\eta v_{2} N_{1}+\eta_{1} k N_{1}^{2} .
$$

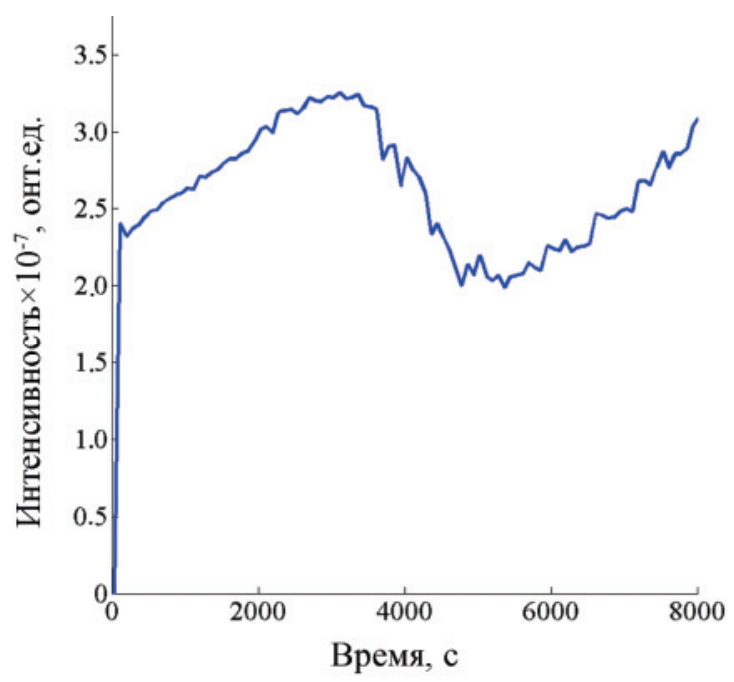

Рис.4. Зависимость интенсивности свечения фосфора $\mathrm{ZnS}-\mathrm{Mn}^{2+}$ от времени при высоких кониентраииях атомов водорода $\geq 10^{13} \mathrm{~cm}^{-3}, T=295 \mathrm{~K}$

Fig. 4. Dependence of the luminescence intensity of phosphorus $\mathrm{ZnS}-\mathrm{Mn}^{2+}$ on time at high concentrations of hydrogen atoms $\geq 10^{13} \mathrm{~cm}^{-3}, T=295 \mathrm{~K}$

Наличие реакции ЛХ проявляется в начальном резком тушении ГХЛ после «выключения» атомов на величину $I_{\mathrm{Pu}}=\eta v_{2} N_{1}, v_{2}=\sigma_{2}(j=0)=0$ (9) и последующем постепенном квадратичном со временем спаде интенсивности свечения ГХЛ

$$
I_{\text {गХ }}=\eta_{1} k N_{1}^{2}=\eta_{1} k N_{1}^{2}(0) /\left(1+k N_{1}(0) t\right)^{2} .
$$

После «выключения» $j=0$ атомов Н интенсивность ГХЛ падает примерно на два порядка (рис. 5) $\left(I_{\mathrm{PU}} / I_{\mathrm{JX}} \approx 100\right)$.

Величины $k N_{1}(0)$ в (10) для различных времен заполнения поверхности атомами водорода приведены в табл. 2 ( $T=306 \mathrm{~K})$.

Таблица 2. Скоростьзатуханиягетерогенной хемилюминесценции Table 2. Speed of heterogeneous chemiluminescent damping

\begin{tabular}{|l|c|c|c|c|c|c|c|}
\hline $\begin{array}{l}\text { Время заполнения поверхности } \\
\text { атомами (мин) } \\
\text { Time of surface filling with } \\
\text { atoms (min) }\end{array}$ & 35 & 80 & 120 & 150 & 200 & 250 & 300 \\
\hline $\begin{array}{l}\text { Скорость затухания гетероген- } \\
\text { ной хемилюминесценции } \\
\text { Heterogeneous chemilumines- } \\
\text { cence decay rate, } k N_{1}(0), \mathrm{c}^{-1}\end{array}$ & 0,2 & 0,29 & 0,31 & 0,3 & 0,35 & 0,36 & 0,37 \\
\hline
\end{tabular}




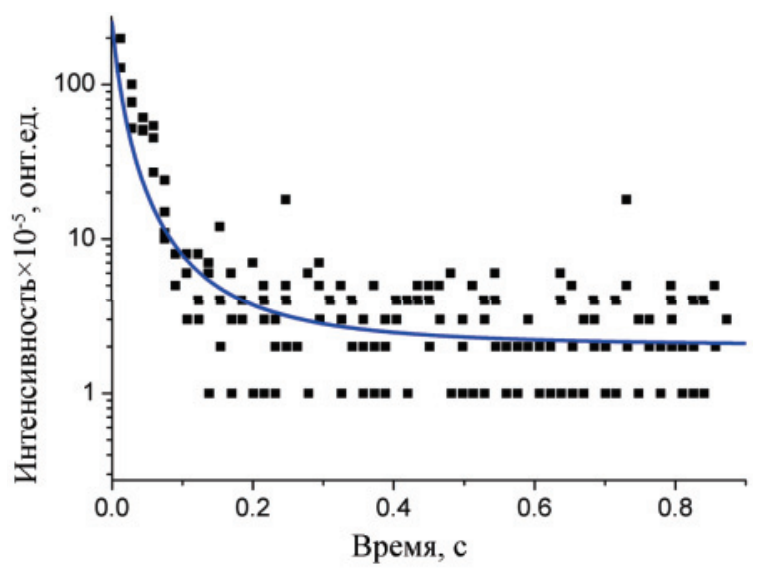

Pис. 5. Затухание свечения фосфора $\mathrm{ZnS}-\mathrm{Mn}^{2+}$ после «выключения» $(j=0)$ атомов водорода. Выключение атомов через 250 мин после начала возбуждения гетерогенной хелилю минесиениии, $\mathrm{T}=306 \mathrm{~K}$

Fig. 5. Damping of phosphorus emission of $\mathrm{ZnS}-\mathrm{Mn}^{2+}$ after the «shutdown» ( $j=0)$ of hydrogen atoms. Shutting of atoms in 250 minutes after start of heterogeneous chemiluminescent excitation, $T=306 \mathrm{~K}$

Предельное заполнение $k N_{1}(0) \leq 10^{15} \mathrm{~cm}^{-2}$, что соответствует $k=(306 K) \approx 4 \cdot 10^{-16} \mathrm{~cm}^{2} \mathrm{c}^{-1}$.

Концентрационно-временные циклы

Интенсивность ГХЛ I пропорциональна скорости ударной рекомбинации атомов

$$
I(j)=B_{1} \sigma_{2} j N_{1}(j) .
$$

При ступенчатом изменении $j$ на $\Delta j$ концентрации адсорбированных частиц не могут измениться мгновенно, поэтому начальный скачок интенсивности $\Delta I$ пропорционален $\Delta j$. Имеем согласно (11)

$$
\frac{\Delta I}{I}=\frac{\Delta j}{j} .
$$

По приращению интенсивности в (12) представляется возможность вычислить новый поток атоMOB

$$
j_{(1)}=j+\Delta j=j\left(1+\frac{\Delta I}{I}\right) .
$$

После выхода релаксационной кривой на стационар $I_{1}$, при потоке атомов $j_{(1)}$ вновь изменим $j_{(1)}$ и по новому приращению интенсивности $\Delta I_{(1)}(13)$ определим

$$
j_{(2)}=j_{(1)}\left(1+\frac{\Delta I_{(1)}}{I_{1}}\right)
$$

и т. д., согласно (13), (14). Люминесцентный метод отличает безынерционность. Запаздывание определяется временем $\tau$ высвечивания возбужденных центров свечения. Даже для запрещенных переходов $\tau \leq 10^{-3}$ с. Это позволяет регистрировать очень быстрые изменения плотностей потоков атомов и весьма просто определять абсолютные значения $j$ и $\Delta j$, если известно абсолютное значение $j$ хотя бы в одной точке.
На рис. 6 приведены релаксационные кинетические кривые ГХ⿰ ${ }_{\mathrm{H}}$, полученные при выключении и включении атомов с изменением плотности потока атомов $j$ на $\Delta j$.

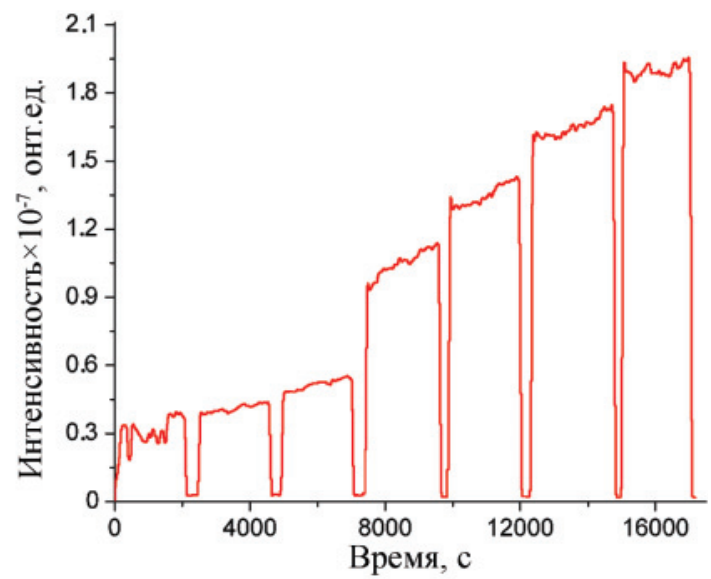

Puc.6. Кинетические кривые люминесценции $\mathrm{ZnS}-\mathrm{Mn}^{2+}$ в атомарном водороде при выключении и включении атомов водорода различной концентрации - темновые паузы. Температура образиа $306 \mathrm{~K}$, давление в разрядной трубке 3,0·10-2 торр, экспозииия фотоэлектронного улножителя 20 мс, диапазон 30 c.

Fig. 6. Kinetic curves of $\mathrm{ZnS}-\mathrm{Mn}^{2+}$ luminescence in atomic hydrogen upon turning off and turning on of hydrogen atoms with various concentrations - «dark» pauses. Sample temperature is $306 \mathrm{~K}$, pressure in the discharge tube is $3,0 \cdot 10^{-2}$ torr, photomultiplier tube exposure is $20 \mathrm{~ms}$, diapason is $30 \mathrm{~s}$

На рис. 7 приведена полученная методом скачков концентраций зависимость интенсивности ГХЛ от плотности потока атомов для малых $j$. Как видно из рис. 7 , интенсивность свечения $\mathrm{ZnS}-\mathrm{Mn}^{2+}$ в атомарном водороде сверхлинейно зависит от концентрации атомов водорода (плотности потока атомов Н) при низких концентрациях атомов, что соответствует ударному (Ридила-Или)

$$
\mathrm{R}+\mathrm{L} \stackrel{v_{1}}{\longrightarrow} \mathrm{RL}, \mathrm{R}+\mathrm{RL} \stackrel{v_{2}}{\longrightarrow} \mathrm{R}_{2}^{\mathrm{v}} \mathrm{L}
$$

механизму возбуждения ГХЛ:

$$
I(j)=\eta N_{0} \frac{\sigma_{1} \sigma_{2}}{v_{-10}} \exp \left(\frac{q_{1}+E_{1}}{k T}\right) j^{2} .
$$

Определим $I(j)$ зависимость стационарной интенсивности ГХЛ от плотности потока атомов с учетом процессов десорбции атомов (I), адсорбции молекул (II), атомно-молекулярного обмена (IV) и диссоциации молекул (V):
I. $\mathrm{R}+\mathrm{L} \underset{v_{-1}}{\stackrel{v_{1}}{\rightleftarrows}} \mathrm{RL}$;
II. $\mathrm{R}+\mathrm{RL} \stackrel{\eta v_{2}}{\longrightarrow} \mathrm{R}_{2} \mathrm{~L}+h v$
III. $\mathrm{R}_{2}+\mathrm{L} \underset{v_{-3}}{\stackrel{v_{3}}{\rightleftarrows}} \mathrm{R}_{2} \mathrm{~L}$;
IV. $\mathrm{R}+\mathrm{R}_{2} \mathrm{~L} \stackrel{v_{4}}{\longrightarrow} \mathrm{R}_{2}+\mathrm{RL}$;
V. $\mathrm{R}_{2} \mathrm{~L}+\mathrm{L} \stackrel{v_{5}}{\longrightarrow} \mathrm{RL}+\mathrm{RL}$. 


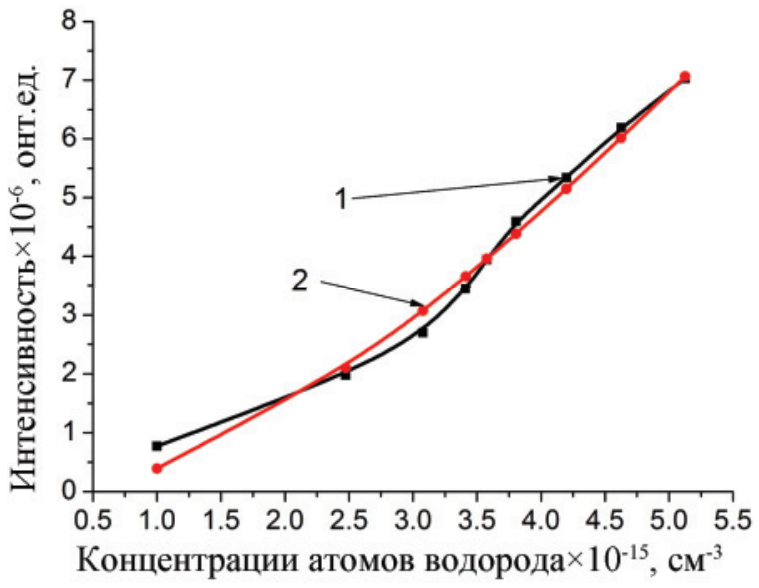

Pис. 7. Зависимость интенсивности гетерогенной хелилюли несиенции $\mathrm{ZnS}-\mathrm{Mn}^{2+}$ в атоларном водороде от концентрации атомов водорода (плотность потока атомов $\left.\sim 10^{15} \mathrm{~cm}^{-2} \mathrm{c}^{-1}\right), T=295 \mathrm{~K}$

Fig. 7. Dependence of intensity of $\mathrm{ZnS}-\mathrm{Mn}^{2+}$ heterogeneous chemiluminescence in atomic hydrogen onhydrogen atoms concentration (atom flux density $\sim 10^{15} \mathrm{~cm}^{-2} \mathrm{~s}^{-1}$ ), $T=295 \mathrm{~K}$

$$
\begin{gathered}
I(j)=\frac{B N_{0} v_{2}\left[v_{1} v_{-3}+\left(v_{1}+v_{3}\right)\left(v_{4}+v_{5}\right)\right]}{\left[\begin{array}{l}
\left(v_{1}+v_{-1}+v_{2}\right)\left(v_{3}+v_{-3}+v_{4}+v_{5}\right)+ \\
+\left(v_{1}-v_{4}-v_{5}\right)\left(v_{2}-v_{3}\right)
\end{array}\right]}= \\
=\frac{\left[\begin{array}{l}
\left.B N_{0} j^{2} \sigma_{2}\left[\sigma_{1} v_{-3}+\sigma_{1} v_{5}+v_{3} \sigma_{4}+\sigma_{4} \sigma_{1} j\right)\right]+ \\
+B N_{0} j \sigma_{2} v_{3} v_{5}
\end{array}\right]}{\left[\begin{array}{l}
j^{2}\left(\sigma_{1} \sigma_{2}+\sigma_{1} \sigma_{4}\right)+j\left[\begin{array}{l}
\sigma_{1}\left(v_{3}+v_{-3}+v_{5}\right)+ \\
+\sigma_{2}\left(v_{3}+v_{-3}\right)+\sigma_{4} v_{-1}
\end{array}\right]+ \\
+v_{-1}\left(v_{3}+v_{-3}+v_{5}\right)+v_{5} v_{3}
\end{array}\right]} .
\end{gathered}
$$

Зависимость стационарной интенсивности ГХЛ плотности потока атомов определяется (1), (2), (15), (16) при $t=\infty\left(r_{1,2}<0\right)$.

$$
\begin{gathered}
I=\frac{B N_{0} \sigma_{1} \sigma_{2} v_{-3} j^{2}}{\sigma_{1} \sigma_{2} j^{2}+\left(\sigma_{1}+\sigma_{2}\right) v_{-3} j+v_{-1} v_{-3}} \approx \\
\approx \frac{B N_{0} \sigma_{1} \sigma_{2} v_{-3} j}{\sigma_{1} \sigma_{2} j+\left(\sigma_{1}+\sigma_{2}\right) v_{-3}} .
\end{gathered}
$$

Интенсивность ГХЛ монотонно нарастает с увеличением $j$ в области высоких $j$ и низких температур, когда в (16), (17) процессы адсорбции преобладают над десорбционными

$$
I(j)=\frac{B N_{0} \sigma_{2}\left[\sigma_{1} v_{-3}+\sigma_{1} v_{5}+v_{3} \sigma_{4}+\sigma_{4} \sigma_{1} j\right]}{\left(\sigma_{1} \sigma_{2}+\sigma_{1} \sigma_{4}\right)} .
$$

Интенсивность ГХЛ достигает насыщения в зависимости от $j(17)$ или может продолжить линейный рост (18) за счет вклада реакции обмена (IV).

Насыщение $I(j)$ при больших потоках атомов связано с задержкой на поверхности молекул, образующихся при ударной рекомбинации атомов явление рекомбинационной блокировки поверхности (рис. 8).

Полученное выражение $I(j)$ (17) качественно верно описывает эксперимент (рис. 8)

$$
I(j)=\frac{j^{2}}{0,42 j^{2}+2,9 j+55}
$$

и позволяет получить оценки величин относительных скоростей реакций

$$
\begin{gathered}
v_{1} \approx 10^{-4} \mathrm{c}^{-1} ; \quad v_{2} \approx 10^{-5} \mathrm{c}^{-1} ; \\
v_{-1}\left(v_{3}+v_{-3}+v_{5}\right) \approx 2 \cdot 10^{-7} \mathrm{c}^{-1} ; v_{5} \approx 0,1\left(v_{1}+v_{2}\right) .
\end{gathered}
$$

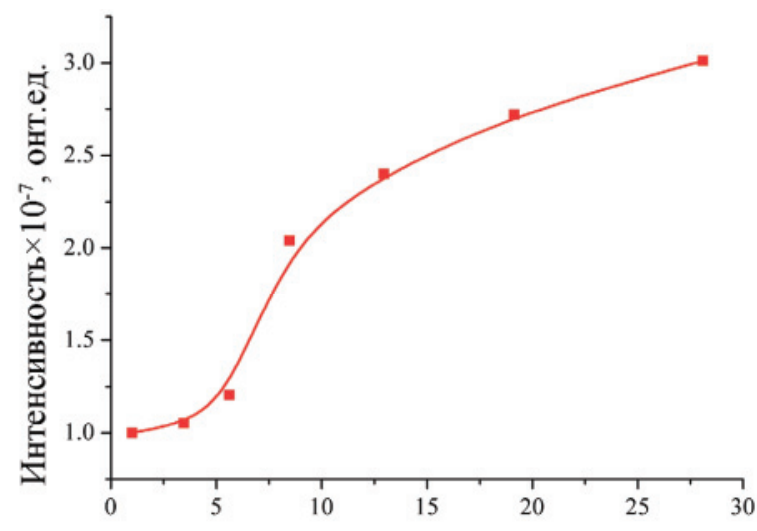

Концентрации атомов водорода $\times 10^{-15}, \mathrm{~cm}^{-3}$

Pис.8. Зависилость стационарной интенсивности гетерогенной хемилюминесиениии люминофора $\mathrm{ZnS}-\mathrm{Mn}^{2+}$ от концентрации атомов водорода. Температура образиа $306 \mathrm{~K}$

Fig. 8. Dependence of stationary intensity of $\mathrm{ZnS}-\mathrm{Mn}^{2+}$ phosphor heterogeneous chemiluminescence on hydrogen atoms concentration. The sample temperature is $306 \mathrm{~K}$

Полученное выражение $I(j)$ (17) качественно верно описывает эксперимент (рис. 8)

$$
I(j)=\frac{j^{2}}{0,42 j^{2}+2,9 j+55}
$$

и позволяет получить оценки величин относительных скоростей реакций

$$
\begin{gathered}
v_{1} \approx 10^{-4} \mathrm{c}^{-1} ; v_{2} \approx 10^{-5} \mathrm{c}^{-1} ; \\
v_{-1}\left(v_{3}+v_{-3}+v_{5}\right) \approx 2 \cdot 10^{-7} \mathrm{c}^{-1} ; v_{5} \approx 0,1\left(v_{1}+v_{2}\right) .
\end{gathered}
$$

Релаксационные кривые интенсивности ГХЛ содержат ту же информацию, что и кинетические кривые разгорания ГХЛ на поверхности, предварительно не заполненной адсорбатом, поскольку кинетический механизм возбуждения и число форм адсорбции остаются неизменными при различных $j$. В дополнение к этому релаксационные кривые содержат новые сведения о вероятности излучательных переходов на поверхности, металлизации поверхности, миграции водорода в объем фосфора и его влиянии на квантовый выход объемных центров свечения и интенсивность фотолюминесценции [46-48].

Люминесцентный метод отличает безынерционность (запаздывание на величину скорости высвечивания возбужденных центров свечения для запрещенных переходов $\tau<10^{-3}$ c), позволяющая регистрировать очень быстрые изменения плотностей потоков атомов, и простота определения абсолютных значений $j$ и $\Delta j$. 


\section{Заключение}

Методы, основанные на явлении гетерогенной хемилюминесценции, дают чувствительный инструмент для изучения химического состава поверхности, әффективности электронных излучательных процессов на поверхности, механизмов переноса энергии и процессов модификации поверхности. Указанные возможности актуальны при реализации плазмохимических методов нанесения защитных, восстановительных, теплозащитных, коррозионностойких, износостойких покрытий для защиты нефтедобывающего оборудования.

Нестационарные люминесцентные методы изучения рекомбинации атомов водорода на поверхности $\mathrm{ZnS}-\mathrm{Mn}^{2+}$ позволили разделить ударный и

\section{СПИСОК ЛИТЕРАТУРЫ}

1. Ивановский В.Н. Коррозия скважинного оборудования и способы защиты от нее // Коррозия. Территория нефтегаз. 2011. - № 1 (18). - C. 18-25.

2. Применение технологий газотермического напыления для решения задач повышения ресурса оборудования и сооружений. Общество с ограниченной ответственностью «Урал - Технологические системы покрытий» // Docslid. 2019. URL https://ural-tsp.ru/wp-content/uploads/2019/01/prezent.pdf (дата обращения 25.11.2019).

3. Гранкин Д.В. Хемилюминесценция облученного УФ-светом кристалла $\mathrm{Zn}_{2} \mathrm{SiO}_{4}-\mathrm{Mn}$ под действием атомов водорода // Журнал физической химии. - 2017. - Т. 92. - № 4. - С. 671-673.

4. Nanomaterial-amplified chemiluminescence systems and their applications in bioassays / Q. Li, L. Zhang, J. Li, C. Lu // TrAC Trends in Analytical Chemistry. - 2011. - V. 30. (2). P. 401-413.

5. Classical oxidant induced chemiluminescence of fluorescent carbon dots / Z. Lin, W. Xue, H. Chen, J.-M. Lin // Chemical Communications. -2012 . - V. 48. - P. 1051-1053.

6. Quantum dots-enhanced chemiluminescence: mechanism and application / H. Chen, L. Lin, H. Li, J.-M. Lin / Coordination Chemistry Reviews. - 2014. - V. 263-264. - P. 86-100.

7. Flow-injection analysis of hydrogen peroxide based on carbon nanospheres catalyzed hydrogen carbonate-hydrogen peroxide chemiluminescent reaction / H. Chen, L. Lin, Z. Lin, C. Lu, G. Guo, J.-M. Lin // Analyst. - 2011. - V. 136. - P. 1957-1964.

8. Plasmonic luminescent core-shell nanocomposites-enhanced chemiluminescence arising from the decomposition of peroxomonosulfite / H. Chen, W. Xue, C. Lu, H. Li, Y. Zheng, J.-M. Lin // Spectrochimica Acta Part A: Molecular and Biomolecular Spectroscopy. - 2013. - V. 116. - P. 355-360.

9. Nanoparticle-assisted chemiluminescence and its applications in analytical chemistry / D.L. Giokas, A.G. Vlessidis, G.Z. Tsogas, N.P. Evmiridis // TrAC Trends in Analytical Chemistry. 2010. - V. 29(10). - P. 1113-1126.

10. Dingkun Zhang, Jin-Ming Lin. Recent development of gas-solid phase chemiluminescence // Journal of Analysis and Testing. 2017. - V. 1 (4). - P. 267-273.

11. One-step enrichment and chemiluminescence detection of sodium dodecyl benzene sulfonate in river water using $\mathrm{Mg}-\mathrm{Al}$-carbonate layered double hydroxides / W. Guan, W. Zhou, D. Han, M. Zhang, C. Lu, J.-M. Lin // Talanta. - 2014. - V. 120. P. 268-273.

12. On-line solid phase extraction of humic acid from environmental water and monitoring with flow-through chemiluminescence / диффузионный процессы рекомбинации атомов водорода и найти доли вкладов ударного и диффузионного механизмов в полную скорость рекомбинации атомов в зависимости от температуры образцов и плотности потока свободных атомов.

Полученные результаты важны для идентификации процессов возбуждения гетерогенной хемилюминесценции, определения элементарных стадий и параметров атомно-молекулярных процессов на поверхности, определения концентрации атомов и состава плазменного потока по интенсивности гетерогенной хемилюминесценции, люминесцентно-активных примесей на поверхности твердых тел, а также для экспресс-управления процессами в плазмохимических системах газтвердое тело.

J. Qu, H. Chen, C. Lu, Z. Wang, J.-M. Lin // Analyst. - 2012. V. 137. - P. 1824-1830

13. Shah S.N., Lin J.M. Recent advances in chemiluminescence based on carbonaceous dots // Advances in Colloid and Interface Science. 2017. - V. 241. - P. 24-36. DOI: 10.1016/j.cis.2017.01.003.

14. Sensitivity and binding kinetics of an ultra-sensitive chemiluminescent enzyme-linked immunosorbent assay at arrays of antibodies / C.I. Tobos, S. Kim, D.M. Rissin, J.M. Johnson, S. Douglas, S. Yan, S. Nie, B. Rice, K.J. Sung, H.D. Sikes, D.C. Duffy // Journal of Immunological Methods. - 2019. - V. 474. 112643 P. 1-13. DOI: 10.1016/j.jim.2019.112643.

15. Flow-based luminescencesensing methods for environmental water analysis / X. Wang, J.M. Lin, M.L. Liu, X.L. Cheng // TrAC Trends in Analytical Chemistry. - 2009. - V. 28 (1). - P. 75-87.

16. Lin Z., Chen H., Lin J.-M. Peroxide induced ultra-weak chemiluminescence and its application in analytical chemistry // Analyst. - 2013. - V. 138 (18). - P. 5182-5193.

17. Preparation of surface imprinting polymer capped Mn-doped ZnS quantum dots and their application for chemiluminescence detection of 4-nitrophenol in tap water / J. Liu, H. Chen, Z. Lin, J.-M. Lin // American Chemical Society. - 2010. - V. 82. P. 7380-7386.

18. Plasmon-assisted enhancement of the ultraweak chemiluminescence using $\mathrm{Cu} / \mathrm{Ni}$ metal nanoparticles / H. Chen, R. Li, H. Li, J.-M. Lin // The Journal of Physical Chemistry C. - 2012. V. 116. - P. 14796-14803.

19. Mestre Y.F., Zamora L.L., Calatayud J.M. Flow-chemiluminescence: a growing modality of pharmaceutical analysis / Luminescence. - 2001. - V. 16. - P. 213-235.

20. Dodeigne C., Thunus L., Lejeune R. Chemiluminescence as diagnostic tool. A review // Talanta. - 2000 - V. 51. - P. 415-439.

21. On the heterogeneous chemiluminescence of $\mathrm{Y}_{2} \mathrm{O}_{2} \mathrm{~S}$ crystal phosphors activated by europium / V.V. Styrov, N.D. Tolmacheva, Y.I. Tyurin, S.K. Shigalugov, V.D. Khoruzhii, Y.A. Sivov, E.Yu. Plotnicova, V.S. Sypchenko // Journal of Surface Investigation. X-ray, Synchrotron and Neutron Techniques. - 2014. V. 8. - P. $1158-1160$

22. Heterogeneous chemiluminescence of crystallophosphor catalysts in the C0+0 mixture / S.K. Shigalugov, Y.I. Tyurin, V.V. Styrov, N.D. Tolmacheva // Kinet Catal. - 2000 - V. 41. - P. 531-537.

23. Гранкин Д.В., Бажин А.И., Гранкин В.П. Генерация высокоэнергетических электронов в металле под действием тепловых атомов водорода и дейтерия из плазмы / / Известия российской академии наук. Серия физическая. - 2017. - Т. 82. - № 2. C. $182-185$.

24. Окисление водорода на палладии: метод хемотоков в нанодиоде шоттки / В.П. Гранкин, Д.В. Гранкин, В.В. Стыров, 
С.В. Симченко, О.А. Гуральник // Журнал физической химии. - 2017. - Т. 91. - № 2. - С. 297-303.

25. Electron accommodation during hydrogen atom adsorption on a juvenile surface of a zinc sulfide single crystal / A.F. Gorbachev, V.V. Styrov, V.M. Tolmachev, Yu.I. Tyurin // Soviet Physics-JETP. - 1986. - V. 64 (1). - P. 99-109.

26. Авторское свидетельство № 1603257 (СССР). Способ измерения адсорбционно-активных газов / А.Ф. Горбачев, В.В. Стыров, В.М. Толмачев, Ю.И. Тюрин // Открытия. Изобретения. 1990. - № 40, - C. 23-28.

27. Shigalugov S.H., Dubrov D.V. Chemiluminescence $\mathrm{Y}_{2} \mathrm{O}_{3}: \mathrm{Bi}^{3+}$ induced by nitrogen oxide // RASAYAN Journal of Chemistry. 2019. - V. 12 (4). - P. 2097-2102.

28. Стыров В.В., Тюрин Ю.И., Шигалугов С.Х. Методы люминесцентного анализа, основанные на явлении гетерогенной хемилюминесценции // Заводская лаборатория. - 1991. - Т. 57. № 11. - C. 1-5.

29. Хемилюминесцентный анализатор озона в атмосферном воздуxe (3.02 П-A). URL: http://www.optec.ru/produktsiya.html? c dept id=16\&c good id=15 (дата обращения 25.11.2019).

30. Microstructures and luminescence behaviors of $\mathrm{Mn}^{2+}$ doped $\mathrm{ZnS}$ nanoparticle clusters with different core shell assembled orders / Chunyan Song, Bing Chen, Yunchao Chen, Xianping Fan // Journal of Alloys and Compounds. - 2013. - V. 590. - P. 546-552. DOI: $10.1016 / j$.jallcom.2013.12.169

31. Murugadoss G., Rajamannan B., Madhusudhanana U. Synthesis and characterization of water-soluble $\mathrm{ZnS}: \mathrm{Mn}^{2+}$ nanocrystals // Chalcogenide Letters. - May 2009 - V. 6 (5). - P. 197-201.

32. Rema Devi B.S., Raveendran R., Vaidyan A.V. Synthesis and characterization of Mn2+-doped ZnS nanoparticles / Synthesis and characterization of nanoparticles. Pramana. - April 2007. - V. 68 (4). P. 87-89. DOI: https://doi.org/10.1007/s12043-007-0068-7

33. Origin of the green photoluminescence from zinc sulfide nanobelts / Changhui Ye, Xiaosheng Fang, Guanghai Li, Lide Zhang // Applied Physics Letters. - 2004. - V. 85. - P. 3035-3037. URL: https://doi.org/10.1063/1.1807018.

34. Vishwakarma H.L., Archana Sharan, Anju Singh. Studies on the synthesis and characterization of nano phosphors for field emission devices // International Journal of Science and Research (IJSR). - June 2014. -V. 3 (6). - P. 219-222. Paper ID: 02013895 .

35. Doped ZnS: Mn nanoparticles obtained by sonochemical synthesis / 0.A. Korotchenkov, A. Cantarero, A.P. Shpak, Yu.A. Kunitskii, A.I. Senkevich, M.O. Borovoy, A.B. Nadtochii // Nanotechnology. - 2005. - V. 16(10). - P. 2033-2038. URL: https://doi.org/ $10.1088 / 0957-4484 / 16 / 10 / 008$.

36. Synthesis and characterization of photoluminescent PVA/ZnS: $\mathrm{Mn}^{2+}$ nanocomposites. / L.Priya // Mapana Journal of Sciences. 2013. - V. 12. - P. 31-37. URL: https://doi.org/10.12723/ mjs.24.4 (дата обращения 25.11.2019).
37. Ru-Shi Liu. Phosphors, up conversion nano particles, quantum dots and their applications. - Berlin, Heidelberg: Springer, 2017.- 593 c. DOI 10.1007/978-3-662-52771-9

38. Шигалугов С.Х. Установка для исследования твердых тел с неравновесными кислородо содержащими газовыми средами люминесцентными методами // Известия Томского политехнического университета. - 2005. - Т. 308. - № 3. - С. 57-64.

39. Tollefson E.L., Le Roy D.J. The reaction of atomic hydrogen with acetylene // The Journal of Chemical Physics. - 1948. - V. 16. № 11. - P. 1055-1062. URL: https://doi.org/10.1063/1.1746724 (дата обращения 25.11.2019).

40. Захарченя В.П., Копылянский А.А. Спектроскопия кристаллов. - Москва: Наука, 1966. - 99 с.

41. Берсукер И.Б. Электронное строение и свойства координационных соединений. - Л.: Химия, 1976. - 352 с.

42. Tanabe Y., Sugano S. On the absorption spectra of complex ions // Journal of the Physical Society of Japan. - 1954. - V. 9. - № 5. P. 753-766.

43. Generation of excited electronic states at the nonmetal surface by the hydrogen atoms beam / Y.I. Tyurin, N.N. Nikitenkov, I.T. Sigfusson, A. Hashhash, Y. Van, N.D. Tolmacheva // International Journal of Hydrogen Energy. - 2017. - V. 42 (17). P. 12448-12457. URL: https://doi.org/10.1016/j.ijhydene.2017.03.058 (дата обращения 25.11.2019).

44. Столяров К.П., Григорьев Н.Н. Введение в люминесцентный анализ неорганических веществ. - Л.: Химия, 1967. - 364 с.

45. Динамика спектров люминесценции кристаллофосфоров ZnS$\mathrm{Cu}, \mathrm{ZnS}-\mathrm{Ag}$ в атомарном водороде / В.Д. Хоружий, Ю.И. Тюрин, В.В. Стыров, Ю.А Сивов // Известия Российской академии наук. Серия физическая. - 2008. - Т. 72. - № 7. C. $978-982$.

46. Эффективность передачи энергии адсорбции и рекомбинации атомов твердому телу при различных механизмах возбуждения / Ю.И. Тюрин, В.Д. Хоружий, С.Х. Шигалугов, Ю.А. Сивов, Т.В. Смекалина // Известия томского политехнического университета. - 2008. - Т. 312. - № 2. - С. 56-65.

47. Nonequilibrium electronic phenomena and the chemical energy accommodation during heterogeneous recombination of atomic hydrogen on the manganese doped willemite / D.V. Grankin, V.P. Grankin, V.V. Styrov, M. Sushchikh // Chemical Physics Letters. - 2016. - V. 647. - P. 145-149. https://doi.org/10.1016/j.cplett.2016.01.049

48. Шигалугов С.Х. Исследование взаимодействия неравновесных кислородосодержащих газовых сред с твердыми телами люминесцентными методами: автореф. дис. ... д-ра физ.-мат. наук. Томск, 2005. - 262 c.

Поступила 02.12.2019 2.

\section{Информация об авторах}

Тюрин Ю.И., доктор физико-математических наук, профессор отделения экспериментальной физики Инженерной школы ядерных технологий Национального исследовательского Томского политехнического университета.

$\boldsymbol{B a н}$ Яомин, аспирант отделения экспериментальной физики Инженерной школы ядерных технологий Национального исследовательского Томского политехнического университета.

Cыnченко B.C., кандидат физико-математических наук, доцент отделения экспериментальной физики Инженерной школы ядерных технологий Национального исследовательского Томского политехнического университета.

Никитенков А.Н., кандидат геолого-минералогических наук, доцент отделения геологии Инженерной школы природных ресурсов Национального исследовательского Томского политехнического университета. 


\title{
METHOD OF FLUORESCENT CONTROL OF PLASMA STRUCTURE AND TREATED SURFACE IN TECHNIQUE OF DOWNHOLE EQUIPMENT ANTICORROSION PROTECTION
}

\author{
Yury I. Tyurin', \\ tyurin@tpu.ru
}

Wang Yaoming',

Vladimir S. Sypchenko',

sypchenkov@mail.ru

\author{
Aleksey N. Niktenkov', \\ nik@tpu.ru
National Research Tomsk Polytechnic University, 30, Lenin avenue, Tomsk, 634050, Russia.

\begin{abstract}
Relevance. Reliability and integrity of oil production equipment, its operation time are provided by a set of measures to combat corrosion, in particular, to use gas-plasma sputtering of protective coatings on designed or restored technology parts. In these technologies, the heterogeneous chemiluminescence phenomenon can serve as an effective method for monitoring plasma composition state and the sputtered surface quality. Heterogeneous chemiluminescence reactions has selectivity and high sensitivity to surface type and excitation gas grade. The use of optical methods to study and control in non-equilibrium gas-solid systems opens up new analytical possibilities in surface physics, chemistry, plasma chemistry, semiconductor and phosphor technology, and in solving environmental problems. Study of adsorption, desorption, dissociation, diffusion, gas particles recombination, defect formation and crystal lattice growth using heterogeneous chemiluminescence phenomenon is an urgent task in condensed matter physics, as the heterogeneous chemiluminescence phenomenon realizes the possibility of selective rapid analysis methods with simple hardware equipment at detection limit of free atoms, radicals, impurities in gas phase and the surface layers composition condensed matter to $10^{-6} \%$ (mol).

The main aim of the research is to study the processes in non-equilibrium systems gas-solid and to determine the interaction based on the registration of characteristics of heterogeneous chemiluminescence; to develop the methods for determining time-dependent interaction parameters of gas-solid using heterogeneous chemiluminescence phenomenon, to monitor the parameters of gaseous medium and the state of condensed matter surface.

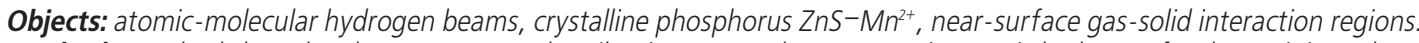

Methods: methods based on heterogeneous chemiluminescence phenomenon in atomic hydrogen for determining adsorption rate and $\mathrm{H}$ atoms recombination, $\mathrm{H}_{2}$ molecules desorption rate, heat of hydrogen atoms desorption from $\mathrm{ZnS}-\mathrm{Mn}^{2+}$ surface. Using the "dark» pause method, the recombination rate of adsorbed atoms was obtained by the Langmuir-Hinshelwood mechanism.

Results. The authors have carried out the comparative study of $\mathrm{ZnS}-\mathrm{Mn}^{2+} /$ luminescence upon light (photoluminescence) and atomic hydrogen (heterogeneous chemiluminescence) excitation. Spectral-kinetic characteristics of luminescence were studied and mechanisms and parameters of interaction of hydrogen atoms with zinc sulfide surface (cross sections, frequency factors, activation energies) based on the kinetic characteristics of heterogeneous chemiluminescence were determined. It is shown that the $\mathrm{ZnS}-\mathrm{Mn}^{2+}$ phosphor can serve as an express sensor reducing plasma component (hydrogen). Heterogeneous chemiluminescence phenomenon is an effective method to control the initial step of modification and composition of solids surface in beam-plasma treatment of materials.
\end{abstract}

Key words:

Oil and gas equipment, corrosion, protective coatings, gas-plasma spraying, photoluminescence, heterogeneous chemiluminescence, surface, hydrogen atoms, non-stationary processes, interactions of hydrogen atoms with the surface.

\section{REFERENCES}

1. Ivanovsky V.N. Korroziya skvazhinnogo oborudovaniya i sposoby zashchity ot nee [Corrosion of downhole equipment and methods of protection against it]. Corrosion. Territory of oil and gas, 2011, no. 1 (18), pp. $18-25$.

2. Primenenie technology gazotermicheskogo napyleniya dlya resheniya zadach povysheniya resursa oborudovaniya i sooruzheny. Obshchestvo s ogranichennoy otvetstvennostyu «Ural - Tekhnologicheskie sistemy pokryty» [Using thermal spraying technology to solve the problems increasing the resource of equipment and structures. Limited Liability Company «Ural - Technological Coating Systems»] 2019. Available at: https://ural-tsp.ru/wp-content/uploads/2019/01/prezent.pdf (accessed 25 November 2019).

3. Grankin D.V. Chemoluminescence of a UV-Irradiated $\mathrm{Zn}_{2} \mathrm{SiO}_{4}-\mathrm{Mn}$ Crystal Exposed to Hydrogen Atoms. Russian Journal of Physical Chemistry, 2008, vol. 92 (4). pp. 816-818.
4. Li Q., Zhang L., Li J., Lu C. Nanomaterial-amplified chemiluminescence systems and their applications in bioassays. TrAC Trends in Analytical Chemistry, 2011, vol. 30 (2), pp. 401-413.

5. Lin Z., Xue W., Chen H., Lin J.-M. Classical oxidant induced chemiluminescence of fluorescent carbon dots. Chemical Communications, 2012, vol. 48, pp. 1051-1053.

6. Chen H., Lin L., Li H., Lin J.-M. Quantum dots-enhanced chemiluminescence: mechanism and application. Coordination Chemistry Reviews, 2014, vol. 263-264, pp. 86-100.

7. Chen H., Lin L., Lin Z., Lu C., Guo G., Lin J.-M. Flow-injection analysis of hydrogen peroxide based on carbon nanospheres catalyzed hydrogen carbonate-hydrogen peroxide chemiluminescent reaction. Analyst, 2011, vol. 136, pp. 1957-1964.

8. Chen H., Xue W., Lu C., Li H., Zheng Y., Lin J.-M. Plasmonic luminescent core-shell nanocomposites-enhanced chemiluminescence arising from the decomposition of peroxomonosulfite. Spec- 
trochimica Acta Part A: Molecular and Biomolecular Spectroscopy, 2013, vol. 116, pp. 355-360.

9. Giokas D.L., Vlessidis A.G., Tsogas G.Z., Evmiridis N.P. Nanoparticle-assisted chemiluminescence and its applications in analytical chemistry. TrAC Trends in Analytical Chemistry, 2010, vol. 29 (10), pp. 1113-1126.

10. Zhang Dingkun, Lin Jin-Ming. Recent development of gas-solid phase chemiluminescence. Journal of Analysis and Testing, 2017, vol. 1 (4), pp. 267-273.

11. Guan W., Zhou W., Han D., Zhang M., Lu C., Lin J.-M. One-step enrichment and chemiluminescence detection of sodium dodecyl benzene sulfonate in river water using $\mathrm{Mg}-\mathrm{Al}$-carbonate layered double hydroxides. Talanta, 2014, vol. 120, pp. 268-273.

12. Qu J., Chen H., Lu C., Wang Z., Lin J.-M. On-line solid phase extraction of humic acid from environmental water and monitoring with flow-through chemiluminescence. Analyst, 2012, vol. 137, pp. 1824-1830.

13. Shah S.N., Lin J.M. Recent advances in chemiluminescence based on carbonaceous dots. Advances in Colloid and Interface Science, 2017, vol. 241, pp. 24-36. DOI: 10.1016/j.cis.2017.01.003.

14. Tobos C.I., Kim S., Rissin D.M., Johnson J.M., Douglas S., Yan S., Nie S., Rice B., Sung K.J., Sikes H.D., Duffy D.C. Sensitivity and binding kinetics of an ultra-sensitive chemiluminescent enzyme-linked immunosorbent assay at arrays of antibodies. Journal of Immunological Methods, 2019, vol. 474, pp. 1-13. DOI: $10.1016 /$ j.jim.2019.112643.

15. Wang X., Lin J.M., Liu M.L., Cheng X.L. Flow-based luminescencesensing methods for environmental water analysis. TrAC Trends in Analytical Chemistry, 2009, vol. 28 (1), pp. 75-87.

16. Lin Z., Chen H., Lin J.-M. Peroxide induced ultra-weak chemiluminescence and its application in analytical chemistry. Analyst, 2013, vol. 138 (18), pp. 5182-5193.

17. Liu J., Chen H., Lin Z., Lin J.-M. Preparation of surface imprinting polymer capped $\mathrm{Mn}$-doped $\mathrm{ZnS}$ quantum dots and their application for chemiluminescence detection of 4-nitrophenol in tap water. American Chemical Society, 2010, vol. 82, pp. 7380-7386.

18. Chen H., Li R., Li H., Lin J.-M.. Plasmon-assisted enhancement of the ultraweak chemiluminescence using $\mathrm{Cu} / \mathrm{Ni}$ metal nanoparticles. The Journal of Physical Chemistry C, 2012, vol. 116, pp. 14796-14803.

19. Mestre Y.F., Zamora L.L., Calatayud J.M. Flow-chemiluminescence: a growing modality of pharmaceutical analysis. Luminescence, 2001, vol. 16, pp. 213-235.

20. Dodeigne C., Thunus L., Lejeune R. Chemiluminescence as diagnostic tool. A review. Talanta, 2000, vol. 51, pp. 415-439.

21. Styrov V.V., Tolmacheva N.D., Tyurin Y.I., Shigalugov S.K., Khoruzhii V.D., Sivov Y.A., Plotnicova E.Yu., Sypchenko V.S.. On the heterogeneous chemiluminescence of $\mathrm{Y}_{2} \mathrm{O}_{2} \mathrm{~S}$ crystal phosphors activated by europium. Journal of Surface Investigation. X-ray, Synchrotron and Neutron Techniques, 2014, vol. 8, pp. 1158-1160.

22. Shigalugov S.K., Tyurin Y.I., Styrov V.V., Tolmacheva N.D. Heterogeneous chemiluminescence of crystallophosphor catalysts in the CO+0 mixture. Kinet Catal., 2000, vol. 41, pp. 531-537.

23. Grankin D.V., Bazhin A.I., Grankin V.P. Generation of high-energy electrons in a metal under the impact of thermal hydrogen atoms and plasma deuterium. Bulletin of the Russian Academy of Sciences: Physics, 2018, vol. 82 (2), pp. 159-162.

24. Grankin D.V., Styrov V.V., Simchenko S.V., Grankin V.P., Gural'nik 0.A. Oxidation of hydrogen on palladium: Chemicurrents in the Schottky nanodiode. Russian Journal of Physical Chemistry A, 2017, vol. 91 (2), pp. 295-300.

25. Gorbachev A.F., Styrov V.V., Tolmachev V.M., Tyurin Yu.I. Electron accommodation during hydrogen atom adsorption on a juvenile surface of a zinc sulfide single crystal. Sov. Phys. JETP, 1986, vol. 64 (1), pp. 99-109.

26. Gorbachev A.F., Styrov V.V., Tolmachev V.M., Tyurin Yu.I. Avtorskoe svidetelstvo no. 1603257 (SSSR). Sposob izmereniya ad- sorbtsionno-aktivnykh gazov [Copyright certificate 1603257 USSR. Method for measuring adsorption-active gases]. Discoveries. Inventions, 1990 , no. 40, pp. 23-28.

27. Shigalugov S.H., Dubrov D.V. Chemiluminescence $\mathrm{Y}_{2} \mathrm{O}_{3}: \mathrm{Bi}^{3+}$ induced by nitrogen oxide. RASAYAN Journal of Chemistry, 2019, vol. 12 (4), pp. 2097-2102.

28. Styrov V.V., Tyurin Yu.I., Shigalugov S.Kh. Metody lyuminestsentnogo analiza, osnovannye na yavlenii geterogennoy khemilyuminestsentsii [Methods of luminescent analysis based on the phenomenon of heterogeneous chemiluminescence]. Factory laboratory, 1991, vol. 57, no. 11, pp. 1-5.

29. Khemilyuminesstentny analizator ozona $v$ atmosfernom vozdukhe [Chemiluminescent analyzer of ozone in atmospheric air (3.02 P-A)]. Available at: http://www.optec.ru/produktsiya.html?c_dept_id=16\&c_good_id=15 (accessed 25 November 2019).

30. Song Chunyan, Chen Bing, Chen Yunchao, Fan Xianping. Microstructures and luminescence behaviors of $\mathrm{Mn}^{2+}$ doped $\mathrm{ZnS}$ nanoparticle clusters with different core shell assembled orders Journal of Alloys and Compounds, 2014, vol. 590, pp. 546-552. DOI: 10.1016/j.jallcom.2013.12.169

31. Murugadoss G., Rajamannan B., Madhusudhanana U. Synthesis and characterization of water-soluble $\mathrm{ZnS}: \mathrm{Mn}^{2+}$ nanocrystals. Chalcogenide Letters, May 2009, vol. 6 (5), pp. 197-201.

32. Rema Devi B.S., Raveendran R., Vaidyan A.V. Synthesis and characterization of $\mathrm{Mn}^{2+}$ doped $\mathrm{ZnS}$ nanoparticles synthesis and characterization of nanoparticles. Pramana Journal of Physics, April 2007, vol. 68 (4), pp. 87-89. D0I: https://doi.org/10.1007/ s12043-007-0068-7

33. Ye Changhui, Fang Xiaosheng, Li Guanghai, Zhang Lide. Origin of the green photoluminescence from zinc sulfide nanobelts. $A p$ plied Physics Letters, 2004, vol. 85, pp. 3035-3037. Available at: https://doi.org/10.1063/1.1807018 (accessed 25 November 2019).

34. Vishwakarma H.L., Archana Sharan, Anju Singh. Studies on the synthesis and characterization of nano phosphors for field emission devices. International Journal of Science and Research (IJSR), June 2014, vol. 3 (6), pp. 219-222. Paper ID: 02013895

35. Korotchenkov 0.A., Cantarero A., Shpak A.P., Kunitskii Yu.A., Senkevich A.I., Borovoy M.O., Nadtochii A.B. Doped ZnS: Mn nanoparticles obtained by sonochemical synthesis. Nanotechnology, 2005. vol. 16, no. 10, pp. 2033-2038. Available at: https://doi. org/10.1088/0957-4484/16/10/008 (accessed 25 November 2019).

36. Priya L. Synthesis and characterization of photoluminescent PVA/ZnS: $\mathrm{Mn}^{2+}$ nanocomposites synthesis and characterization of photoluminescent. Mapana Journal of Sciences, 2013, vol. 12, pp. 31-37. Available at: https://doi.org/10.1088/0957-4484/ 16/10/008 (accessed 25 November 2019).

37. Ru-Shi Liu. Phosphors, up conversion nano particles, quantum dots and their applications. Berlin, Heidelberg, Springer, 2017. 593 p. DOI 10.1007/978-3-662-52771-9

38. Shigalugov S.Kh. Installation for studying interaction of solids with nonequilibrium hydrogen-containing gaseous media by luminescent techniques. Bulletin of the Tomsk Polytechnic University, 2005, vol. 308, no. 3, pp. 57-64. In Rus.

39. Tollefson E.L., Le Roy D.J. The Reaction of Atomic Hydrogen with Acetylene. The Journal of Chemical Physics, 1948, vol. 16, no. 11, pp. 1055-1062. Available at: https://doi.org/10.1063/ 1.1746724 (accessed 25 November 2019).

40. Zakharchenya V.P., Kopylyansky A.A. Spektroskopiya kristallov [Spectroscopy of crystals]. Moscow, Nauka Publ., 1966. 99 p.

41. Bersuker I.B. Elektronnoe stroenie i svoystva koordinatsionnykh soyedineniy [Electronic structure and properties of coordination compounds]. Leningrad, Khimiya Publ., 1976. 352 p.

42. Tanabe Y., Sugano S. On the absorption spectra of complex ions. Journal of the Physical Society of Japan, 1954, vol. 9, no. 5, pp. 766-779. 
43. Tyurin Y.I., Nikitenkov N.N., Sigfusson I.T., Hashhash A., Van Y., Tolmacheva N.D. Generation of excited electronic states at the nonmetal surface by the hydrogen atoms beam. International Journal of Hydrogen Energy, 2017, vol. 42 (17), pp. 12448-12457. Available at: https://doi.org/10.1016/j.ijhydene.2017.03.058 (accessed 25 November 2019).

44. Stolyarov K.P., Grigoriev N.N. Vvedenie v lyuminestsentny analiz neorganicheskikh veshchestv [Introduction to the luminescent analysis of inorganic substances]. Leningrad, Khimiya Publ., 1967. $364 \mathrm{p}$

45. Khoruzhii V.D., Tyurin Yu.I., Styrov V.V., Sivov Yu.A. Dynamics of the luminescence spectra of $\mathrm{ZnS}-\mathrm{Cu}$ and $\mathrm{ZnS}-\mathrm{Ag}$ crystalline phosphors in atomic hydrogen. Izvestiya Rossiiskoi Akademii Nauk. Seriya Fizicheskaya, 2008, vol. 72, no. 7, pp. 978-982.

46. Tyurin Yu.I., Khoruzhiy V.D., Shigalugov S.H., Sivov Yu.A., Smekalina T.V. Efficiency of transfer of adsorption energy and atom recombination to a solid at various excitation mechanisms.
Bulletin of the Russian Academy of Sciences: Physics, 2012, vol. 76 (6), pp. 687-689.

47. Grankin D.V., Grankin V.P., Styrov V.V., Sushchikh M. Nonequilibrium electronic phenomena and the chemical energy accommodation during heterogeneous recombination of atomic hydrogen on the manganese doped willemite. Chemical Physics Letters, 2016, vol. 647, pp. 145-149. Available at: https://doi.org/ 10.1016/j.cplett.2016.01.049 (accessed 25 November 2019).

48. Shigalugov S.Kh. Issledovanie vzaimodeystviya neravnovesnykh kislorodosoderzhashchikh gazovykh sred s tverdymi telami lyuminestsentnymi metodami. Avtoreferat Dis. Doct. nauk [Luminescence of solids surface excited in heterogeneous reactions with oxygen and oxygen-containing particles. Dr. Diss. Abstract]. Tomsk, 2005. $36 \mathrm{p}$.

Received: 2 December 2019.

\section{Information about the authors}

Yury I. Tyurin, Dr. Sc., professor, National Research Tomsk Polytechnic University.

Wang Yaoming, Graduate student, National Research Tomsk Polytechnic University.

Vladimir S. Sypchenko, Cand Sc., associate professor, National Research Tomsk Polytechnic University.

Aleksey N. Niktenkov, Cand Sc., associate professor, National Research Tomsk Polytechnic University. 